\title{
Impact of Miniaturized Fixed-Bias Multi-Needle Langmuir Probes on CubeSats
}

\author{
Sigvald Marholm*, Richard Marchand ${ }^{\dagger}$, Diako Darian ${ }^{\ddagger}$, Wojciech J. Miloch*, and Mikael Mortensen ${ }^{\ddagger}$ \\ ${ }^{*}$ Department of Physics, University of Oslo, P.O. Box 1048 Blindern, N-0316 Oslo, Norway ${ }^{\dagger}$ Department of \\ Physics, University of Alberta, Edmonton AB, Canada $\ddagger$ Department of Mathematics, University of Oslo, P.O. Box \\ 1048 Blindern, N-0316 Oslo, Norway
}

\begin{abstract}
Kinetic simulation results are presented to study the response of multi-Needle Langmuir Probes of the type used on many satellites. Simulations of isolated probes are used to parametrize the current collected as a function of voltage for a set of densities and temperatures of relevance to Earth ionosphere. These simulations also serve to assess the validity of analytic results obtained from the orbit motion limited (OML) theory used in recent studies. Computed probe characteristics are then fitted with empirical scaling laws and used to account for electron current collected by needle probes on a typical triple CubeSat. These fits are then used to determine the impact of the probes and guards on the spacecraft floating potential for a nominal configuration of bias voltages, over the plasma parameters of interest. In order for the probes to work as intended, they must operate at a positive potential with respect to the ambient plasma. However, results show that for the cases considered, the spacecraft floating potential is so low that the probe with the lowest voltage becomes negative. Possible solutions are examined and proposed to ensure that all probes remain at a positive voltage with respect to surrounding plasma.
\end{abstract}

Index Terms-Langmuir probes, floating potential, finitelength effects, Low Earth Orbit, sheath, CubeSat.

\section{INTRODUCTION}

$\mathbf{L}$ ANGMUIR probes are the instrument of choice in many laboratory and space plasma experiments. They are easy to build, and there exist simple theoretical models that can be used to derive physical quantities, such as the electron density and temperature, from the measurements.

For thin cylindrical Langmuir probes (needle probes), the orbital motion limited (OML) theory predicts that two probes with fixed bias voltage are sufficient to eliminate any temperature dependence and compute the electron density. While having a single probe with a swept bias voltage is also possible, the sweeping time, which is of the order of seconds, reduces the spatial resolution of space-borne instruments [1]. For this reason, the miniaturized fixed-bias multi-Needle Langmuir Probes (m-NLP) have spurred interest in the nano satellite community, and several CubeSats have been equipped with a set of four needle probes (4-NLP) [2].

However, the theory used in the interpretation of these probes makes several assumptions, most importantly that the probes are infinitely long. The probes on todays CubeSats are typically $25 \mathrm{~mm}$ long, and probes of 50 and even $100 \mathrm{~mm}$ have been considered in order to improve performance. According to some experiments this should give significantly improved results [3], while others conclude that finite length effects are significant even for probes in excess of 50 Debye lengths $\left(\lambda_{D}\right)$ [4]. None of the suggested lengths are sufficient to overcome the $50 \lambda_{D}$ barrier for typical ionospheric conditions, where the Debye length may range from about 2 to $12 \mathrm{~mm}$.

The second issue we address is the effect the probes have on the floating potential. Fixed-bias Langmuir probes are supposed to operate at a positive voltage with respect to the ambient plasma. It is a common concern that the spacecraft may be so negatively charged that this condition is not satisfied, especially for a small spacecraft where the positively biased Langmuir probes may collect a considerable amount of charge compared to positive charges that can be collected by the surface of the spacecraft.

In the present work we address both issues with numerical, Particle-In-Cell (PIC) simulations. We first study in detail the probe currents and probe environment for $50 \mathrm{~mm}$ long probes and provide curve-fits to their current-voltage characteristics. We then continue with simulations of a three unit CubeSat to study how a 4-NLP instrument affects the floating potential of the satellite. We finally simulate how altered bias voltages and geometries of the 4-NLP instrument can be used to maintain an appropriate floating potential of the spacecraft. This is an extension of the work in [5].

The layout of the paper is as follows: In Section II we present the background theory on measurements using fixedbias probes. Simulations of Langmuir probes as well as CubeSat floating potentials are presented in Section III. First the methods and results are presented, and then the results are discussed in subsequent subsections. In Section IV we suggest some improvements based on the shortcomings discovered. Finally the conclusion follows in Section V.

\section{BACKGROUND}

When a conducting object is fixed at a positive voltage $V$ with respect to the ambient plasma, it attracts electrons and repels positive ions, and thus collects a negative electric current $I$. Mott-Smith and Langmuir derived analytic expressions for this current using what is today known as orbital motion limited (OML) theory. The results can be summarized as [6], [3]

$$
I=-n e C A \sqrt{\frac{k T}{2 \pi m}}\left(1+\frac{e V}{k T}\right)^{\beta}
$$


TABLE I

OML PARAMETERs APPEARING IN (1) FOR THREe GeOMEtries

\begin{tabular}{lccl}
\hline & $C$ & $A$ & $\beta$ \\
\hline Planar & 1 & & 0 \\
Cylindrical & $2 / \sqrt{\pi}$ & $2 \pi r l$ & 0.5 \\
Spherical & 1 & $4 \pi r^{2}$ & 1 \\
\hline
\end{tabular}

Here, $r$ and $l$ are probe radius and length, respectively.

Here, $-e, m, n$ and $T$ are the charge, mass, density and temperature of the electron, while $k$ is Boltzmann's constant. The expression is valid for both planar, cylindrical and spherical objects, with the correct values for the constants $C, A$, and $\beta$ listed in Table I. $A$ is simply the surface area of the object. The expression is valid for a plasma that is 1) collisionless, 2) non-drifting Maxwellian and 3) nonmagnetized. These assumptions are justified in [1] and will not be further investigated here. It is further required that 4 ) the ion collected current is much smaller than the electron collected current. This is true for typical ionospheric conditions with ions drifting at approximately $7500 \mathrm{~m} / \mathrm{s}, 5$ ) that the object is not affected by other, nearby objects, and 6) $\mathrm{eV} / k T>0$ or, for cylindrical objects, $e V / k T \gtrsim 2$. As for the probe geometry, it is assumed that 7$)$ the probe is very thin $\left(r \ll \lambda_{D}\right)$ and, for cylindrical probes, 8$)$ very long $\left(l \gg \lambda_{D}\right)$. The m-NLP probes on CubeSats typically have a radius of $0.255 \mathrm{~mm} \mathrm{[2]} \mathrm{such} \mathrm{that}$ $r / \lambda_{D}<0.2$ for the Debye lengths considered. According to numerical simulations by Laframboise [7], finite-radius effects are not significant even for $r / \lambda_{D}=1$, so this assumption is valid. The assumptions $e V / k T \gtrsim 2$ and $l \gg \lambda_{D}$ will be discussed further in what follows. The vicinity of other objects is only briefly considered.

Langmuir probes use current-voltage characteristics (1) to infer electron density and/or temperature from current/voltage measurements. In particular, for a fixed-bias Langmuir probe $p$, the probe is fixed at a bias voltage $V_{0 p}$ with respect to the spacecraft, which itself is at an unknown floating potential $V_{0}$ with respect to the ambient plasma, i.e. the probe potential is $V_{p}=V_{0}+V_{0 p}$. It was observed by Jacobsen et al. [1] that when a cylindrical probe is used, the OML theory predicts that the current squared splits into a temperature-dependent part, and a voltage-dependent part:

$$
I_{p}^{2}=\frac{(n e C A)^{2}}{2 \pi m}\left[k T+e\left(V_{0}+V_{0 p}\right)\right] .
$$

By subtracting the current squared for two such probes at different biases $V_{01}$ and $V_{02}$, the unknown temperature as well as the unknown floating potential are eliminated, and one can rearrange the result to get the electron density:

$$
n^{2}=\frac{2 \pi m}{(C A)^{2} e^{3}}\left(\frac{I_{2}^{2}-I_{1}^{2}}{V_{02}-V_{01}}\right) .
$$

However, if the OML theory does not hold because one or more of the assumptions listed above are not satisfied, this expression may be inaccurate. In fact, finite-length effects are significant for todays CubeSat probes of $25 \mathrm{~mm}$ length (roughly $2.5 \lambda_{D}$ for typical Debye lengths of $10 \mathrm{~mm}$ ), though the literature is diverse as to how bad the finite-length effects are, and how long probes must be to overcome them. For example, Hoang et al. [3] and Johanning et al. [8] agree that 5-10 Debye lengths strongly diminish the finite-length effects, whereas Hoskinson et al. [4] finds that even 50 Debye lengths is not enough to justify the use of OML theory.

On the other hand, if one imagines the probe shortening until $l \ll \lambda_{D}$ it will appear as just a tiny, spherical speck compared to the Debye length, and the OML theory for a spherical probe, with $\beta=1$ instead of 0.5 , becomes valid. Since (1) is valid for very short and very long probes, it is reasonable to expect the current-voltage characteristic to be of the same shape for finite-length probes, but with different values for $\beta$ and the product $C A$ ( $C$ and $A$ are unseparable). If one assumes $e V / k T \gg 1$, the OML equation (1) takes the form

$$
I \approx-a V^{\beta}
$$

For finite lengths, the coefficients $a$ and $\beta$ can not be inferred from OML theory, but must be determined by fitting it to numerical simulations. This makes (4) an empirical relation, and it is to be applied only within the range of parameters in which it is constructed, and for which uncertainties are known to be small. This relation will be used when studying the floating potential of the CubeSat.

\section{Simulations of Probe Characteristics AND CubeSat Floating Potential}

In the following, we use unstructured Particle-In-Cell (PIC) simulations from the program PTetra [9], [10] to study the impact $\mathrm{m}$-NLPs have on the floating potential of small spacecrafts, in particular a triple CubeSat. However, due to the very fine scale of the probes compared to the satellite body, it is computationally prohibitive to do a full simulation of a satellite with probes. Instead, we first do separate simulations of a probe at various fixed voltages with respect to the ambient plasma, and from the measured collected currents we do a curve-fit to the semi-empirical power law (4). Next, we simulate the spacecraft without probes, but for each timestep we add the charge $\Delta Q=I \Delta t$ collected by the probes during the time-step $\Delta t$ to the satellite, where $I$ is given by the empirical fit. The charge collected by the probes will then depend on the spacecraft potential, since the probes have a fixed bias with respect to the spacecraft. Simulations are carried out in time until a steady state is reached, from which the floating potential is obtained.

The approach outlined above relies on the assumption that the fields due to the probe and the rest of the spacecraft do not interact sufficiently to significantly affect the net collected current of the spacecraft. This assumption is supported by the fact that the probes and the guards (the part in direct contact with the probes) have the same voltage, and moreover, that the bulk of the spacecraft is separated from the probe and has a much larger collection area.

In order to cover the typical environment experienced by a small satellite in a (circular) Low Earth Orbit at $400 \mathrm{~km}$ altitude, we have chosen to simulate a range of three electron densities and three electron temperatures. The parameters are 
TABLE II

Debye Lengths $[\mathrm{mm}]$ for the Simulation Parameters

\begin{tabular}{cc|ccc}
\hline & & \multicolumn{3}{|c}{$n\left[10^{11} \mathrm{~m}^{-3}\right]$} \\
& & 1.0 & 3.5 & 12.0 \\
\hline$\vec{\Xi}$ & 0.08 & 6.6 & $3.6^{*}$ & $1.9^{*}$ \\
$\stackrel{\vec{\Xi}}{*}$ & 0.17 & 9.7 & 5.2 & $2.8^{*}$ \\
$\stackrel{5}{*}$ & 0.26 & 12.0 & 6.4 & 3.5 \\
\hline
\end{tabular}

* CubeSat simulations with these parameters use an extra fine mesh.

TABLE III

$e V / k T$ FOR THE TEMPERATURE RANGe CONSIDERED IN THE SiMULATIONS

\begin{tabular}{|c|c|c|c|c|c|}
\hline & \multicolumn{4}{|c|}{$V[\mathrm{~V}]$} \\
\hline & & 1.0 & 3.0 & 5.0 & 7.0 \\
\hline$>$ & 0.08 & 12.5 & 37.5 & 62.5 & 87.5 \\
\hline e & 0.17 & 5.9 & 17.6 & 29.4 & 41.2 \\
\hline 3 & 0.26 & 3.8 & 11.5 & 19.2 & 26.9 \\
\hline
\end{tabular}

shown in Table II along with the resulting Debye lengths $\lambda_{D}=$ $\sqrt{\varepsilon_{0} k T / e^{2} n}\left(\varepsilon_{0}\right.$ is the vacuum permittivity). The ions are all taken to be $\mathrm{O}^{+}$ions of the same density and temperature as the electrons. The ionospheric plasma drift velocity relative to the spacecraft is assumed to be $7672 \mathrm{~m} / \mathrm{s}$ (i.e., roughly the orbital velocity).

\section{A. Probe Simulations}

In order to do the current-voltage curve-fits, simulations were carried out for each density and temperature, with several probe voltages ranging from 1 to 7 volts with respect to the ambient plasma. Representative $e V / k T$ ratios are shown in Table III. The geometry, created using Gmsh [11], is shown in Fig. 1, and the simulations were initialized with up to 10 million particles per species, such that each simulation particle corresponded to 1-5 physical particles. All simulations ran for more than $4 \mu \mathrm{s}$, with a time-step of at most $0.51 \mathrm{~ns}$, and the currents were averaged by an Exponential Moving Average (EMA) filter with relaxation time $\tau=0.1 \mu$ s before the last datapoint is taken as the steady-state current. The currents include both ion and electron collection. EMA filtering of a discrete time-series $\left\{x_{0}, x_{1}, \ldots, x_{N}\right\}$ is achieved recursively, through

$$
\begin{aligned}
\bar{x}_{0} & =x_{0}, \\
\bar{x}_{i} & =w_{i} \bar{x}_{i-1}+\left(1-w_{i}\right) x_{i}, \quad i=1, \ldots, N
\end{aligned}
$$

where $w_{i}=\exp \left(-\left(t_{i}-t_{i-1}\right) / \tau\right)$, and $t_{i}$ is the time at time-step $i$. As an example, Fig. 2 shows the unfiltered and EMA-filtered time-evolution of current collected by probes at different voltages in a plasma with $n=1.0 \times 10^{11} \mathrm{~m}^{-3}$ and $k T=0.08 \mathrm{eV}$. The steady-state currents are then used to determine the fitting coefficients $a$ and $\beta$ in (4).

\section{B. Results of Probe Simulations}

Iso-contours at steady-state for the sheath formation around the probes are shown in Fig. 3, and fitting coefficients for the power law (4) are presented in Table IV. The simulated

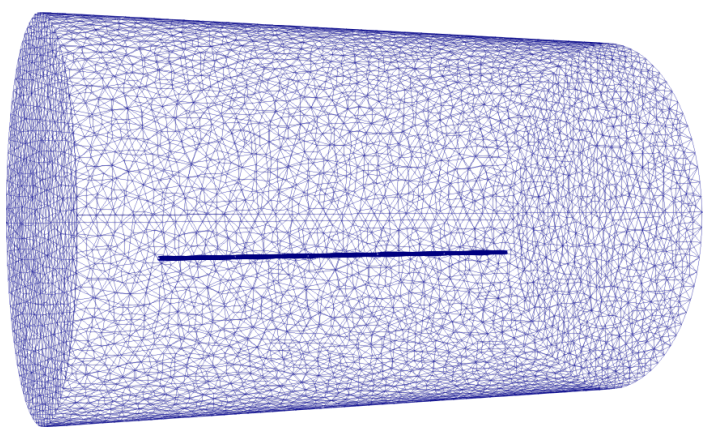

Fig. 1. Simulation geometry of a $50 \mathrm{~mm}$ cylindrical Langmuir probe of $0.255 \mathrm{~mm}$ radius. The cylindrical outer boundary has a radius of $25 \mathrm{~mm}$ and is $80 \mathrm{~mm}$ long. The probe is slightly off-centered (by $5 \mathrm{~mm}$ ) inside the outer boundary to allow more space for the wake to develop (upwards in the figure). The mesh has a resolution of $2 \mathrm{~mm}$ on the outer boundary and $0.085 \mathrm{~mm}$ on the probe, resulting in 464245 tetrahedral cells.

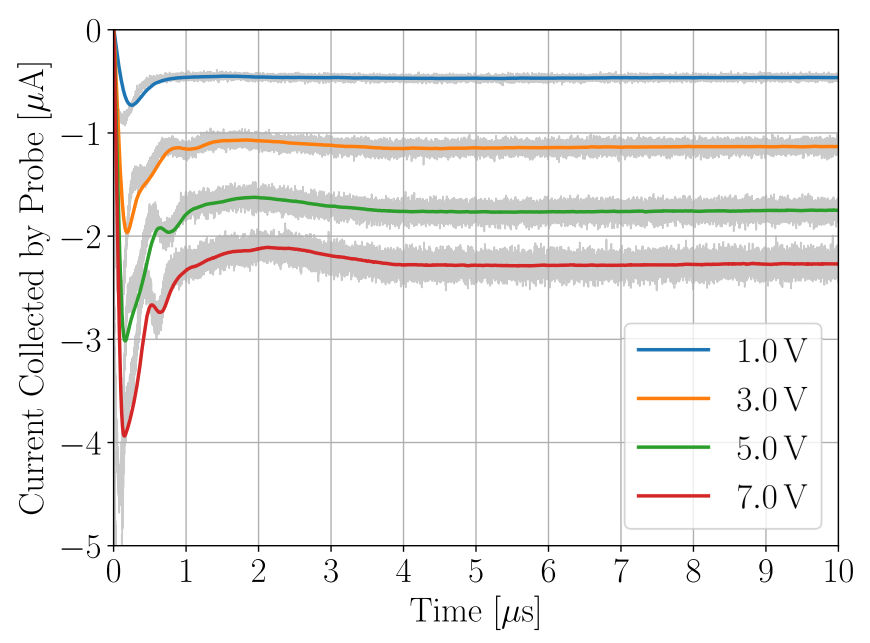

Fig. 2. Evolution of the current collected by probes at different voltages with respect to an ambient plasma with $n=1.0 \times 10^{11} \mathrm{~m}^{-3}$ and $k T=0.08 \mathrm{eV}$. The gray curves are the raw, unfiltered currents, whereas the colored ones are EMA-filtered with a relaxation time of $0.1 \mu \mathrm{s}$.

collected probe currents are on average $1.1 \%$ off the curvefit and at most $3.4 \%$ off. For reference the steady-state probe currents are listed in Appendix A.

\section{CubeSat Simulations}

The geometry for the satellite simulations is shown in Fig. 4. In addition to the $30 \times 10 \times 10 \mathrm{~cm}^{3}$ body of the spacecraft, four booms are included, which are used to separate the needle probes from the body in order to avoid measuring perturbations caused by the spacecraft itself. Each boom is supporting a shorter cylindrical segment, the guard, which is itself attached to the probe [2]. The plane of the probes is $3 \mathrm{~cm}$ ahead of the satellite body, and each boom starts $2 \sqrt{2} \mathrm{~cm}$ diagonally off-center. See Table V for further dimensions. All booms are grounded to the satellite body, whereas the guards are biased to the same potential as the probe they support, as in actual CubeSats. This is intended to reduce fringe effects at the connection points. Only the currents collected by the probes are used in measurements. The four probes are biased at $2.5,4.0,5.5$ and 7.0 volts with respect to the spacecraft 


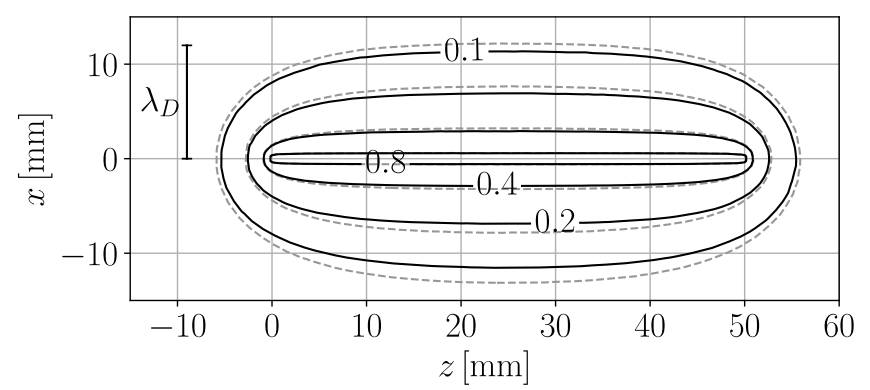

(a) $n=1.0 \times 10^{11} \mathrm{~m}^{-3}, k T=0.26 \mathrm{eV}$

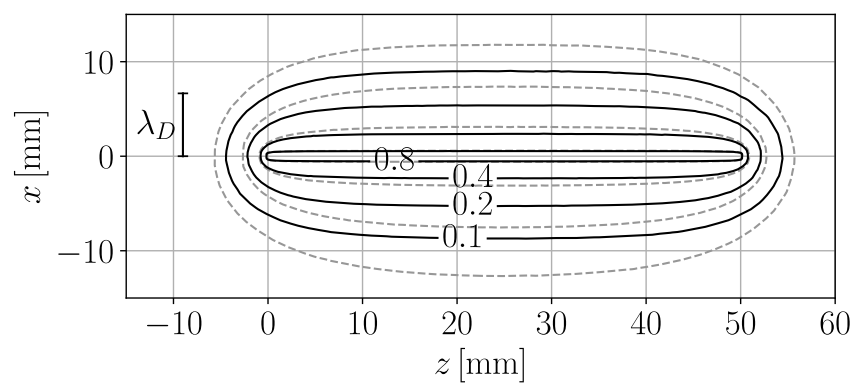

(b) $n=1.0 \times 10^{11} \mathrm{~m}^{-3}, k T=0.08 \mathrm{eV}$

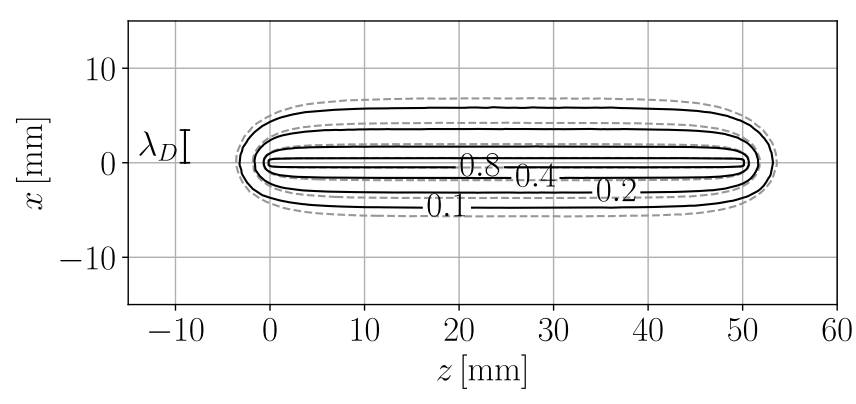

(c) $n=12.0 \times 10^{11} \mathrm{~m}^{-3}, k T=0.26 \mathrm{eV}$

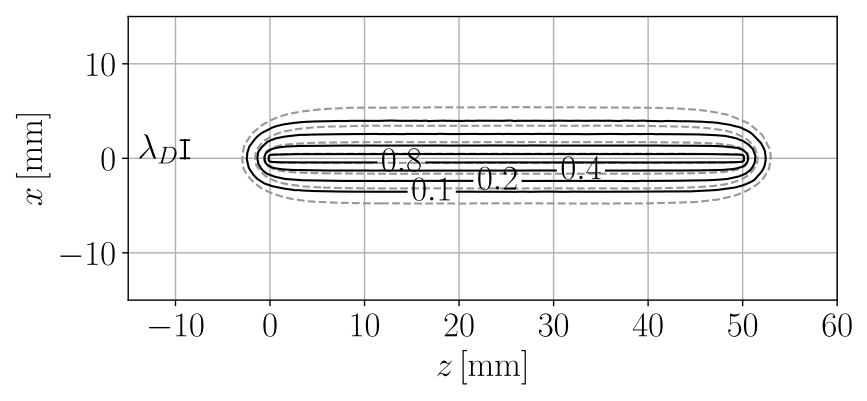

(d) $n=12.0 \times 10^{11} \mathrm{~m}^{-3}, k T=0.08 \mathrm{eV}$

Fig. 3. Illustration of equipotentials in the electric sheath surrounding a cylindrical probe at $1 \mathrm{~V}$ (solid lines) and $7 \mathrm{~V}$ (dashed lines) with respect to the ambient plasma. The contours show the potential in fractions of the probe potential. For reference the Debye length is also illustrated. The drift-velocity (and hence also the wake) is in the $-x$ direction.
TABLE IV

FitTING COEFFICIENTS APPEARING IN (4) FOR THE CURRENTS COLlected By A $50 \mathrm{~mm}$ CYLINDRICAL PROBE

\begin{tabular}{|c|c|c|c|c|}
\hline \multicolumn{5}{|c|}{$a\left[\mu \mathrm{A} / \mathrm{V}^{\beta}\right]$} \\
\hline & & \multicolumn{3}{|c|}{$n\left[10^{11} \mathrm{~m}^{-3}\right]$} \\
\hline & & 1.0 & 3.5 & 12.0 \\
\hline & 0.08 & 0.462 & 1.465 & 4.555 \\
\hline D & 0.17 & 0.415 & 1.372 & 4.316 \\
\hline 5 & 0.26 & 0.392 & 1.323 & 4.244 \\
\hline
\end{tabular}

\begin{tabular}{|c|c|c|c|c|}
\hline \multicolumn{5}{|c|}{$\beta$ (dimensionless) } \\
\hline & & \multicolumn{3}{|c|}{$n\left[10^{11} \mathrm{~m}^{-3}\right]$} \\
\hline & & 1.0 & 3.5 & 12.0 \\
\hline & 0.08 & 0.820 & 0.812 & 0.789 \\
\hline$\stackrel{0}{5}$ & 0.17 & 0.786 & 0.780 & 0.758 \\
\hline s & 0.26 & 0.755 & 0.751 & 0.728 \\
\hline
\end{tabular}

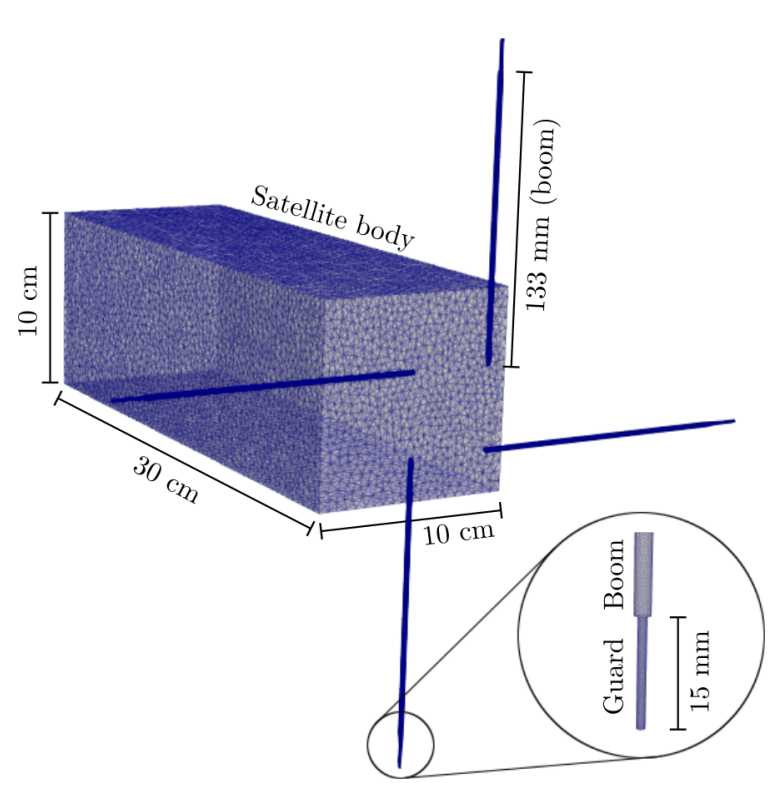

Fig. 4. Illustration of the spacecraft geometry considered in the simulations, with four booms and guards on the ram side, parallel to one of the smaller faces of the satellite. The guard is nearly indistinguishable from the boom unless it is zoomed into. Needle probes are not included in the geometry and the currents that they collect are accounted for in the simulations using the scaling law (4).

respectively, whereas the potential of the spacecraft is selfconsistently determined from charges accumulated by all parts of the assembly, as well as currents derived from the power law for the probes. Since ion-collecting probes collect a negligible current, no charge is added for probes which have turned negative.

The meshes were created using Gmsh [11], with a resolution of $5 \mathrm{~mm}$ on the outer boundary and the satellite body, and

TABLE V

Geometric Parameters of the Boom-Probe System

\begin{tabular}{lccc}
\hline & Boom & Guard & Probe \\
\hline Length [mm] & 133 & 15 & 50 \\
Radius [mm] & 1.1 & 0.595 & 0.255 \\
\hline
\end{tabular}


a fifth of the radius on all cylindrical parts. This results in roughly 4.5 million tetrahedral cells. An extra fine mesh had to be used for simulating the conditions with the shortest Debye length (c.f. Table II) in order to properly resolve the Debye length. The domain of this finer mesh had a resolution of $3 \mathrm{~mm}$ on the outer boundary and satellite body, and a third of the radius for the cylindrical parts. The outer boundary also had to be made somewhat smaller in order to reduce the computational cost to a feasible level. However, the boundary is still at least $5 \mathrm{~cm}$ away from the spacecraft in any direction. This mesh consists of roughly 8.5 million tetrahedral cells. See also Fig. 7. In all cases, the simulations are initialized with 140 million simulation particles of each species. Each simulation particle then corresponds to $70-1000$ physical particles, depending on the parameters of the simulation.

Since the simulations are very time-consuming, most of them have not reached a sufficiently steady state. To correct for this we perform a non-linear least squares curve-fit of the latter half of the simulated spacecraft potential to the function

$$
f(t)=f_{0}+f_{1} \exp \left(-\frac{t}{\nu}\right),
$$

with $f_{0}, f_{1}$ and $\nu$ as fitting coefficients. $f_{0}$ is then the steadystate value, or floating potential. That the first part of the simulated spacecraft potential is excluded from the fitting is important, because it contains faster transient exponential and oscillatory components which would degrade the fit. These are simulation artefacts due to unphysical initial conditions, namely a uniform plasma containing a spacecraft with zero charge.

For the sake of illustration, in Fig. 5 we consider the evolution of the spacecraft potential for the simulation that has reached furthest into the steady-state region. The spacecraft potential reaches $-3.65 \mathrm{~V}$ at the end of this simulation, but if the simulation had been stopped after $20 \mu \mathrm{s}$, the spacecraft potential would only have been $-3.46 \mathrm{~V}$. The curve-fit predicts $-3.66 \mathrm{~V}$ both when applied after $20 \mu \mathrm{s}$ and after the end of the simulation.

All simulations have run for at least $20 \mu \mathrm{s}$. The latter half is without significant transients for all simulations.

Currents collected by the guards are also recorded. In order to reduce noise, they are first EMA-filtered with a relaxation time of $0.1 \mu \mathrm{s}$ before their last half is curve-fitted to (6) similarly to the spacecraft potential. For illustration see Fig. 6.

\section{Results of CubeSat Simulations}

Floating potentials for the satellite are listed in Table VI, for the simulated densities and temperatures. Currents collected by the four probes and guards are also given in Table VII. The probe currents are obtained from the power law (4) for the floating potentials in Table VI, whereas the currents collected by the guards are obtained directly from the kinetic simulations described in Section III-C.

\section{E. Discussion of Probe Simulations}

The curve-fits of the simulation results of a $50 \mathrm{~mm}$ probe to (4) lead to $\beta \sim 0.8$, which is in clear disagreement with $\beta \sim$

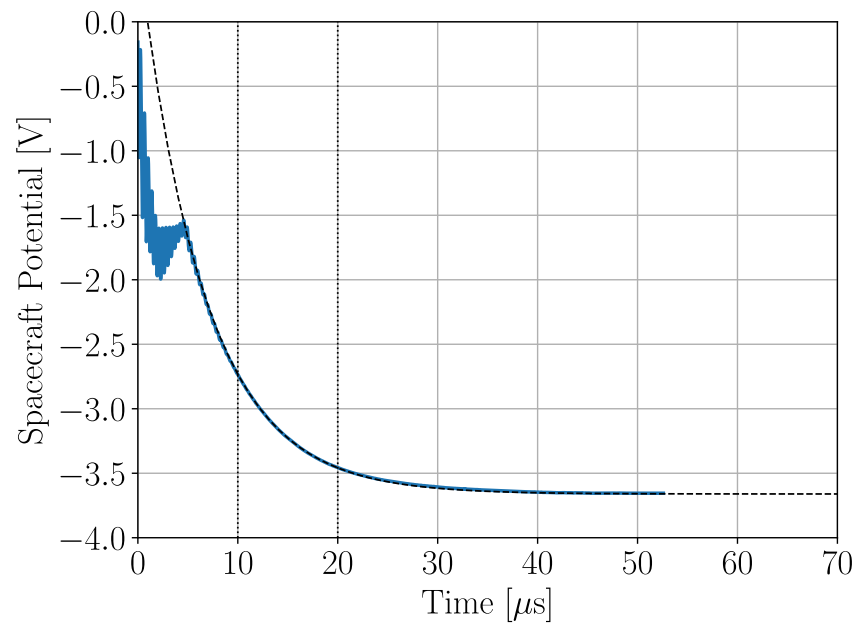

Fig. 5. Evolution of spacecraft potential for $n=12.0 \times 10^{11} \mathrm{~m}^{-3}$ and $k T=0.26 \mathrm{eV}$. The dashed line shows the curve-fit of the region between $10 \mu \mathrm{s}$ and $20 \mu \mathrm{s}$ to (6), used to better determine the steady-state potential.

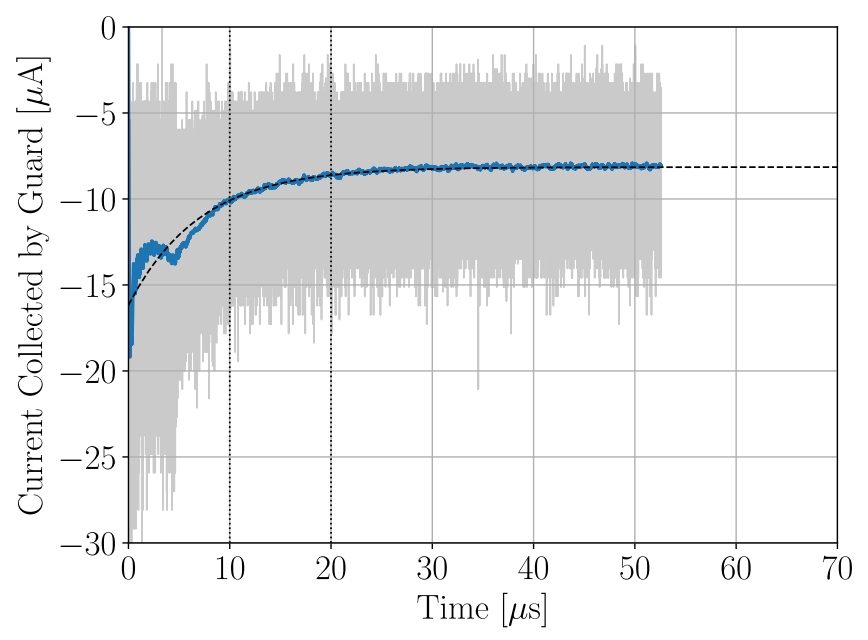

Fig. 6. Evolution of the current collected by the guard with $7.0 \mathrm{~V}$ bias in a plasma with $n=12.0 \times 10^{11} \mathrm{~m}^{-3}$ and $k T=0.26 \mathrm{eV}$. The gray curve are the raw, unfiltered current, whereas the blue one is EMA-filtered with a relaxation time of $0.1 \mu \mathrm{s}$. The dashed line shows a curve-fit of the region between $10 \mu \mathrm{s}$ and $20 \mu \mathrm{s}$ to (6).

TABLE VI

Floating CubeS at Potentials [V] for the Densities and TEMPERATURES CONSIDERED

\begin{tabular}{|c|c|c|c|c|}
\hline & \multicolumn{3}{|c|}{$n\left[10^{11} \mathrm{~m}^{-3}\right]$} \\
\hline & & 1.0 & 3.5 & 12.0 \\
\hline & 0.08 & -3.53 & -3.88 & -4.06 \\
\hline & 0.17 & -3.21 & -3.61 & -3.83 \\
\hline s & 0.26 & -2.99 & -3.41 & -3.66 \\
\hline
\end{tabular}




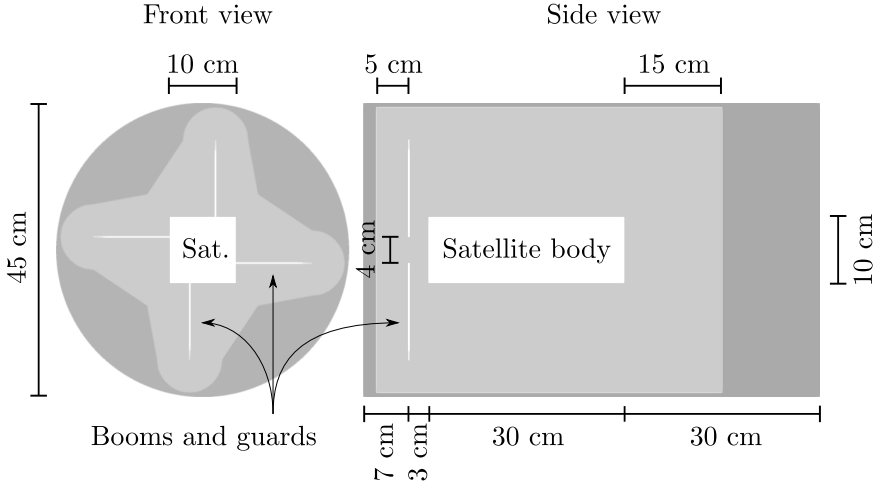

Fig. 7. The boundary of the CubeSat simulation domain is a prism extending in the direction of the satellite body. For the coarse mesh it is cylindrical with a diameter of $45 \mathrm{~cm}$ (dark gray). It extends $30 \mathrm{~cm}$ downstream of the satellite and $7 \mathrm{~cm}$ upstream of the plane of the probes, which is again $3 \mathrm{~cm}$ upstream of the satellite body. The cross section of the fine mesh (light gray) is not as simple, but is at any point at least $5 \mathrm{~cm}$ away from the objects of interest. It extends $15 \mathrm{~cm}$ downstream of the satellite and $5 \mathrm{~cm}$ upstream of the probes. The probes themselves are not part of the geometry.

TABLE VII

Currents Collected by Probes and Guards $[\mu \mathrm{A}]$ Biased at 2.5, 4.0, 5.5 AND 7.0 VOLTS (Listed IN THAT ORDER). 0 INDICATES THAT A Probe's Potential has Become Negative And That it Therefore IS SET TO COLlect EXACTLY ZERO CURRENT.

\begin{tabular}{|c|c|c|c|c|c|c|c|}
\hline & & \multicolumn{6}{|c|}{$n\left[10^{11} \mathrm{~m}^{-3}\right]$} \\
\hline & & \multicolumn{2}{|c|}{1.0} & \multicolumn{2}{|c|}{3.5} & \multicolumn{2}{|c|}{12.0} \\
\hline & & probes & guards & probes & guards & probes & guards \\
\hline \multirow{12}{*}{$\frac{\overrightarrow{0}}{E}$} & \multirow{4}{*}{0.08} & 0 & 0.00 & 0 & 0.01 & 0 & 0.03 \\
\hline & & -0.25 & -0.12 & -0.26 & -0.16 & 0 & -0.20 \\
\hline & & -0.81 & -0.50 & -2.17 & -1.54 & -6.07 & -4.50 \\
\hline & & -1.28 & -0.93 & -3.69 & -2.72 & -10.7 & -8.55 \\
\hline & \multirow{4}{*}{0.17} & 0 & 0.00 & 0 & 0.01 & 0 & 0.04 \\
\hline & & -0.34 & -0.19 & -0.66 & -0.42 & -1.12 & -1.05 \\
\hline & & -0.80 & -0.53 & -2.25 & -1.57 & -6.37 & -4.48 \\
\hline & & -1.18 & -0.90 & -3.56 & -2.61 & -10.3 & -8.06 \\
\hline & \multirow{4}{*}{0.26} & 0 & -0.01 & 0 & 0.00 & 0 & 0.02 \\
\hline & & -0.39 & -0.24 & -0.89 & -0.58 & -1.94 & -1.60 \\
\hline & & -0.79 & -0.56 & -2.30 & -1.62 & -6.62 & -4.92 \\
\hline & & -1.12 & -0.88 & -3.45 & -2.63 & -10.2 & -8.10 \\
\hline
\end{tabular}

0.5 found in [3] and [8]. However, our results do agree well with those of Hoskinson et al. [4], who suggest the empirical relation $\beta=1-0.5 \exp \left(-8 \lambda_{D} / l\right)$ when the radius is much smaller than the Debye length. With this relation the value of $\beta$ should range between 0.63 and 0.92 for the Debye lengths considered here.

Actual m-NLPs have a guard that reduces edge-effects on one end of the probe. In the simulations we have recorded the current collected for each centimeter of the probe individually. This gives us some means for assessing the edge effects. If the guard eliminates edge effects on one end of the probe completely, one would expect the two centimeter-long segments closest to the guard to collect the same amount of current as the middle section. An estimated collected current is then obtained from the simulations by counting the current from the middle segment three times, while ignoring the two segments closest to one of the ends. Performing the curve-fits of these currents to (4) does not alter the values of $a$ and $\beta$ by more than $7 \%$ and $4 \%$, respectively, compared to the values in Table IV.

The implication here is that when $\beta$ is significantly different from 0.5 , there remains a temperature dependence in $I_{2}^{2}-I_{1}^{2}$, and (3) cannot be used to accurately determine the density. In such cases, it may be preferable to consider an alternative approach such as the one presented in [3].

It is also interesting to consider the shape of the sheath, given that previous estimates of end-effects have made assumptions on this shape. For instance, Jaeger et al. [12] assumed the sheath to be cylindrical plus hemispherical on the ends. Considering Fig. 3, this is evidently not the case for the lower densities. The edge-effects extend far inwards on the probe, and the sheath looks more like a prolate spheroid than a cylinder. For the higher densities, when the probe is much larger than the Debye length, the assumption of a cylindrical plus hemispherical sheath is better approached. This shows that Jaeger's assumption should be limited to probes that are much longer than the Debye length.

The sheath also expands as the voltage increases, and the ratio of the long axis to the shorter axes (aspect ratio) decreases, i.e., it becomes less prolate. For the higher temperature this change in shape of equipotentials is less pronounced, while for the lower temperature the difference is more pronounced. This is explained by a much larger normalized voltage $e V / k T$ for the low temperature case. For the higher temperatures $\mathrm{eV} / \mathrm{kT}$ never exceeds roughly 25 , while for the lower temperature this ratio is between 10 and 90 (c.f. Table III). We conclude that as $e V / k T$ increases, the sheath becomes relatively larger, and less prolate, but as long as $e V / k T \lesssim 25$ the sheath is roughly fixed. This agrees well with the results by Choinière [13], [14].

Table IV shows that for a given temperature, $\beta$ decreases as the density increases. This to be expected since, under this condition, higher densities correspond to smaller values of the Debye length, and the fact that larger ratios of the probe length to the Debye length lead to collected currents being closer to those predicted in the OML approximation for an infinite probe; that is, to $\beta$ closer to 0.5 . It is interesting to note however, that for a given density, as the temperature increases, leading to an increase in the Debye length, $\beta$ decreases. This counter-intuitive result, on the sole basis of the probe length to Debye length ratio, can be understood by the fact that the Debye length is not the only factor affecting the effective collection cross section of the probe. Indeed, referring to (1), the effective radial probe cross section, including the thermal speed factor, is also affected by the temperature. In the idealized OML approximation $(\beta=0.5)$, when $e V / k T \gg 1$, temperature variations have a negligible effect. In a non-ideal situation, for a finite length probe with $\beta>0.5$ however, an increase in $T$ with a fixed density and potential, leads to a decrease in the effective cross section. This in turn tends to increase the ratio of length to effective radial collection cross section, thus reducing the relative contribution of end effects, and lowering $\beta$. We note that while (4) with the coefficients in Table IV fit well with data in Table IX, it is not meant to be applicable beyond the range of plasma parameters and probe voltages considered. In particular, it should not be extrapolated 
to large temperatures for which the assumption $e V / k T \gg 1$ made in (4), would break down.

It should be noted that the semi-empirical power law (4) was derived assuming $e V / k T \gg 1$, which is not very well satisfied for the $1.0 \mathrm{~V}$ probe simulations. Nevertheless, the low fitting error indicate that it is a reasonable approximation for further use in the simulations of the CubeSat floating potential.

\section{F. Discussion of CubeSat Simulations}

Equation (1) assumes $e V / k T \gtrsim 2$ which means that the probe voltage should be at least $0.16-0.52$ volts with respect to the ambient plasma for the temperatures considered. At the very least the probe should be collecting electrons, i.e., be positive with respect to ambient plasma [3]. In the absence of Langmuir probes one would expect an object such as a CubeSat to have a floating potential of roughly $-0.5 \mathrm{~V}$ to $-1.0 \mathrm{~V}$. Using a simplified model we have simulated the floating potential of a triple CubeSat with electron-collecting probes. The probes are accounted for indirectly through the power law (4), and the model does not account for 1) every component of the spacecraft, e.g., the solar panels, 2) the Earth's magnetic flux density, 3) day-side photoelectron emission and 4) possible magnetic connectivity between probes and other spacecraft components. Nonetheless the model should capture the effect the probes have on the floating potential of the spacecraft.

Probes with a voltage between $0 \mathrm{~V}$ and $1 \mathrm{~V}$ fall outside the range the power law (4) was fitted for (probes with negative potential collect no current in the simulations). That a probe merely passes through this region in the transient phase can be disregarded, since it will re-adjust. However, from Table VI it can be seen that in many cases the CubeSat floating potential is between $-3 \mathrm{~V}$ and $-4 \mathrm{~V}$, therebye leaving the probe biased at $4 \mathrm{~V}$, between $0 \mathrm{~V}$ and $1 \mathrm{~V}$ with respect to the plasma at steadystate. Even though the current collected by these probes may be less accurate, they collect far less current than the guards and the other probes. Considering for instance the case of $n=12 \times 10^{11} \mathrm{~m}^{-3}$ and $k T=0.17 \mathrm{eV}$, the lowest probe is predicted to collect $-1.12 \mu \mathrm{A}$. By comparison, the guards and all the other probes combined collect $-30.3 \mu \mathrm{A}$. All probes are below the upper limit of $7 \mathrm{~V}$ with respect to the plasma. This supports the power law (4) as a valid approximation in this study.

Unfortunately, the simulations show that due to the extra current collected by the probes and guards, the floating CubeSat potential is always below $-2.5 \mathrm{~V}$, and consequentially, the probe with the lowest bias voltage $(2.5 \mathrm{~V})$ is negative with respect to the ambient plasma. With todays $25 \mathrm{~mm}$ probes, the lower probe is often excluded from the analysis due to suspicion that it is negative [3]. For $50 \mathrm{~mm}$ probes, even the second lowest probe is compromised, as the floating potential may even go down to $-4.1 \mathrm{~V}$.

It is also interesting to note that the spacecraft floating potential becomes less negative as the temperature increases. This may seem counter intuitive since, in the absence of photoelectrons or secondary electron emission, the floating potential of a satellite is generally negative and proportional in absolute value, to the temperature. This dependence on $T$ of the floating potential is a result of the decrease in electron current collected by the probes as $T$ increases, c.f. (1).

\section{Remedies of the Floating Potential}

Several possible solutions to the floating potential problem come to mind: 1) The most obvious is perhaps not to increase the length of the probes beyond the current $25 \mathrm{~mm}$. 2) From Table VII the guard is seen to collect a significant fraction of the electron current. This then leads to the question as to whether it could be made shorter, which would then lead to a reduced overall collected electron current. 3) Another possibility is to use a different set of probe biases. One could try to raise the biases such that the probe voltages become positive. However, higher probe voltages lead to an increase in collected currents, which in turn lowers the potential of spacecraft and probes. On the other hand, lower biases may lead to less collected currents, and higher spacecraft potential, but will it be enough to make the probes voltages higher despite the lower bias? Since only the lower biased probe(s) tend to become negative with respect to background plasma, a solution could be to reduce the difference between the higher and lower bias voltages in such a manner that the net collected current remains roughly the same. That is, the sum of (4) over all probes is roughly the same, given the same floating potential.

Four more cases were simulated to study the effectiveness of such changes in probe geometry and bias voltages. For the middle case, with a density of $n=3.5 \times 10^{11} \mathrm{~m}^{-3}$ and temperature of $k T=0.17 \mathrm{eV}$, we have carried out simulations with: 1) the guard shortened to $5 \mathrm{~mm}, 2$ ) the bias voltages of the four probes (and guards) set to 3.6, 4.4, 5.2 and 6.0 volts, and 3 ) both measures combined. In addition, we have simulated the effect of both measures combined assuming a density of $n=12.0 \times 10^{11} \mathrm{~m}^{-3}$ and temperature $k T=0.08 \mathrm{eV}$, for which the spacecraft potential is the lowest.

\section{A. Results}

The floating potential with these three proposed improvements are listed in Table VIII. For reference, simulations without the proposed improvements (from Section III) are also included. The lower probe voltages are also listed in order to ease the comparison. For completeness, the currents collected by the guards is listed in Appendix A. The probe currents can be derived from (4), should they be of interest.

\section{B. Discussion}

Considering the simulations with $n=3.5 \times 10^{11} \mathrm{~m}^{-3}$ and $k T=0.17 \mathrm{eV}$ in Table VIII, it is seen that shortening the guard (without changing the biases) helps somewhat in raising the probe potentials, although not enough to make the lower one positive. Changing the biases, without changing the guard, does not change the floating potential significantly. This is not surprising, given that the bias voltages were chosen to make the net collected probe current unchanged. Some change is of course unavoidable, since the law for current collected by 
TABLE VIII

Simulated Floating Potentials With a Reduced DifFERENCE Between the LARgest AND Lowest Probe Biases, AND/OR WITH Shorter GuARDs. Two RePrESENTATIVE DENSITIES AND Temperatures HaVE BeEN AsSUMED IN THEsE COMPARISONS.

\begin{tabular}{cccccc}
\hline $\begin{array}{c}\text { Density } \\
{\left[10^{11} \mathrm{~m}^{-3}\right]}\end{array}$ & $\begin{array}{c}\text { Temp. } \\
{[\mathrm{eV}]}\end{array}$ & $\begin{array}{c}\text { Guard } \\
{[\mathrm{mm}]}\end{array}$ & $\begin{array}{c}\text { Biases } \\
{[\mathrm{V}]}\end{array}$ & \multicolumn{2}{c}{$\begin{array}{c}\text { Voltages }[\mathrm{V}] \\
\text { satellite }\end{array}$} \\
\hline \multirow{3}{*}{3.5} & & 15 & $2.5,4.0,5.5,7.0$ & -3.61 & -1.11 \\
& 0.17 & 5 & $2.5,4.0,5.5,7.0$ & -3.26 & -0.76 \\
& & 15 & $3.6,4.4,5.2,6.0$ & -3.49 & 0.11 \\
& & 5 & $3.6,4.4,5.2,6.0$ & -3.22 & 0.38 \\
\hline \multirow{2}{*}{12.0} & 0.08 & 15 & $2.5,4.0,5.5,7.0$ & -4.06 & -1.56 \\
& & 5 & $3.6,4.4,5.2,6.0$ & -3.59 & 0.01 \\
\hline
\end{tabular}

the probes (4) do not account for the current collected by the guards. Although the spacecraft floating potential is not significantly affected, the higher bias of the lower probe is just enough to make it positive. In fact, reducing the difference between the higher and lower bias voltages was more effective than shortening the guard.

The best effect is of course achieved when taking both measures together. When both the guard is shortened and the span of the bias voltages is reduced, the lower probe potential barely dips below zero volt even for the density and temperature yielding the lowest floating potential. Clearly the second lower probe is no longer compromised. These results suggest that the lower probe voltage might be increased even more by using even smaller increments in bias voltages; for example using 3.5, 4.0, 4.5 and 5.0 volts for the four probes.

In practice of course there is a lower limit to the voltage difference between probes, as too small increments would result in unacceptably low signal to noise (or uncertainty) ratio from the measurements. As argued above, too large increments on the other hand, would lead to the lower bias probes being negative. The determination of the optimal increment, for a given instrument is beyond the scope of this work, but it should be considered in the design of future CubeSat missions using fixed bias $\mathrm{m}-\mathrm{NLPs}$.

We note that it is not known how much a shorter guard would affect the performance of the instrument. A study on how long the guards must be to be effective would be considered in the future.

\section{CONCLUSION}

In recent years the use of m-NLPs on small satellites have gained in popularity due to their high spatial resolution and simple analysis following from the OML theory [2]. However, the OML theory assumes the probes to be much longer than the Debye length, and todays $25 \mathrm{~mm}$ are not sufficient to justify this assumption. As a response, longer probes have been suggested [3]. We have carried out PIC simulations of four $50 \mathrm{~mm}$ probes on a triple CubeSat. Our simulations show that probes and guards will collect a considerable amount of electrons due to their positive potential with respect to the ambient plasma, thereby reducing the floating potential of the spacecraft. If the floating potential becomes too negative, the lower biased probe(s) may also become negative and they can no longer be used in analyses based on OML theory for positive probes. Even with todays $25 \mathrm{~mm}$ probes it is common practice to exclude the probe with the lowest bias voltage from the analysis due to suspicion that it has become negative [3]. With the larger collecting surface of $50 \mathrm{~mm}$ probes, the simulations confirm that the lower probe will indeed become negative, and moreover, that the second lowest probe is at risk of becoming negative as well.

We have demonstrated that it is possible to avoid a negative potential by using a shorter guard which will collect less current, and by reducing the difference between the higher and lower bias voltages. Specifically, using a guard of $5 \mathrm{~mm}$ and bias voltages of 3.6, 4.4, 5.2 and 6.0 volts increases the lower probe voltage by roughly $1.5 \mathrm{~V}$ compared to using a guard of $15 \mathrm{~mm}$ and bias voltages of $2.5,4.0,5.5$, and 7.0 volts, enough to stay positive in many cases, though not quite for the temperature and density yielding the lowest floating potential. A better effect may be achieved by reducing the voltage increment somewhat more, for instance by using probe biases of 3.5, 4.0, 4.5 and 5.0 volts.

Our results also show, in agreement with Hoskinson et al. [4], that even $50 \mathrm{~mm}$ probes are not enough to justify the use of OML theory. Since lengthening the probe even more will result in an even lower floating potential, and is nevertheless impractical, one should look for alternative methods of inferring the density, and possibly also temperature and floating potential from these probes. Instead of trying to combat finite-length effects, we suggest trying to take them into account in the analysis by means of simulations. One approach could be to make fits of simulated currents to analytical equations containing density, temperature and floating potential. Given at least three current measurements, this could then be inverted. Another approach would be an attempt at multi-variate regression from a four-tuple of currents to the physical quantities of interest. This would avoid the inversion problem. Future studies along these ideas are needed in order to construct more robust and accurate algorithms to interpret $\mathrm{m}$-NLP measurements in terms of space plasma environment parameters.

\section{ACKNOWLEDGMENT}

This work was supported in part by the 4DSpace Strategic Research Initiative at the University of Oslo, the Natural Sciences and Engineering Research Council of Canada and the Research Council of Norway grant no. 240000. Simulation results presented here made use of the Compute Canada computing infrastructure as well as the Abel Cluster, owned by the University of Oslo and Uninett/Sigma2, and operated by the Department for Research Computing at USIT, the University of Oslo IT-department, Notur project number NN9299K.

\section{APPENDIX A \\ ADDitional TABLES}

For completeness this appendix contains the table of steadystate collected probe currents, Table IX, and in Table X, the guard current for the remedies presented in Section IV. 
TABLE IX

Currents Collected $[\mu \mathrm{A}]$ By a $50 \mathrm{~mm}$ Cylindrical Probe.

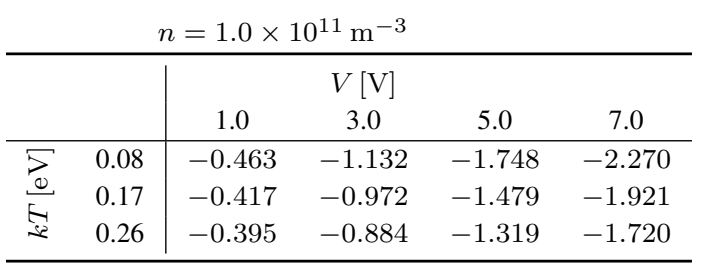

\begin{tabular}{|c|c|c|c|c|}
\hline \multicolumn{5}{|c|}{$n=3.5 \times 10^{11} \mathrm{~m}^{-3}$} \\
\hline & & \multicolumn{3}{|c|}{$V[\mathrm{~V}]$} \\
\hline & & 1.0 & 4.0 & 7.0 \\
\hline 7 & 0.08 & -1.471 & -4.443 & -7.203 \\
\hline$\frac{e}{6}$ & 0.17 & -1.308 & -3.961 & -6.353 \\
\hline 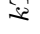 & 0.26 & -1.332 & -3.661 & -5.800 \\
\hline
\end{tabular}

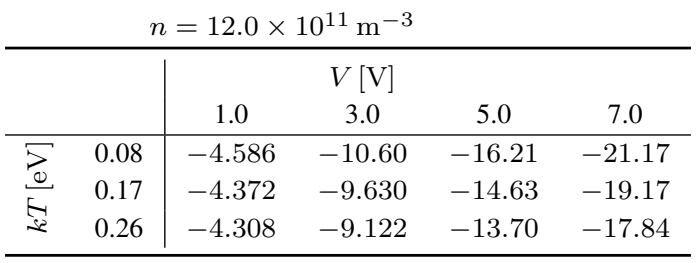

TABLE $X$

Collected Guard Currents for a Reduced Difference Between THE Highest AND Lowest Probe Biases AND/OR With SHORTER GUARDS

\begin{tabular}{|c|c|c|c|c|}
\hline $\begin{array}{c}\text { Density } \\
{\left[10^{11} \mathrm{~m}^{-3}\right]}\end{array}$ & $\begin{array}{c}\text { Temperature } \\
{[\mathrm{eV}]}\end{array}$ & $\begin{array}{l}\text { Guard } \\
{[\mathrm{mm}]}\end{array}$ & $\begin{array}{c}\text { Bias } \\
{[\mathrm{V}]}\end{array}$ & $\begin{array}{c}\text { Guard Current } \\
{[\mu \mathrm{A}]}\end{array}$ \\
\hline \multirow{4}{*}{3.5} & \multirow{4}{*}{0.17} & \multirow{4}{*}{5} & 2.5 & 0.00 \\
\hline & & & 4.0 & -0.16 \\
\hline & & & 5.5 & -0.60 \\
\hline & & & 7.0 & -1.15 \\
\hline \multirow{4}{*}{3.5} & \multirow{4}{*}{0.17} & \multirow{4}{*}{15} & 3.6 & -0.22 \\
\hline & & & 4.4 & -0.82 \\
\hline & & & 5.2 & -1.42 \\
\hline & & & 6.0 & -2.01 \\
\hline \multirow{4}{*}{3.5} & \multirow{4}{*}{0.17} & \multirow{4}{*}{5} & 3.6 & -0.09 \\
\hline & & & 4.4 & -0.27 \\
\hline & & & 5.2 & -0.53 \\
\hline & & & 6.0 & -0.79 \\
\hline \multirow{4}{*}{12.0} & \multirow{4}{*}{0.08} & \multirow{4}{*}{5} & 3.6 & -0.02 \\
\hline & & & 4.4 & -0.81 \\
\hline & & & 5.2 & -1.84 \\
\hline & & & 6.0 & -2.65 \\
\hline
\end{tabular}

\section{REFERENCES}

[1] K. S. Jacobsen, A. Pedersen, J. I. Moen, and T. A. Bekkeng, "A new langmuir probe concept for rapid sampling of space plasma electron density," Measurement Science and Technology, vol. 21, no. 8, p. 085902, 2010. [Online]. Available: http://stacks.iop.org/0957$0233 / 21 / \mathrm{i}=8 / \mathrm{a}=085902$

[2] T. A. Bekkeng, "Development of a miniaturized multi-needle langmuir probe system for in-situ measurements of electron density and spacecraft floating potential," Ph.D. dissertation, University of Oslo, 2017.

[3] H. Hoang, K. Røed, T. A. Bekkeng, J. I. Moen, A. Spicher, L. B. N. Clausen, W. J. Miloch, E. Trondsen, and A. Pedersen, "A study of data analysis techniques for the multi-needle langmuir probe," Measurement
Science and Technology, vol. 29, no. 6, p. 065906, 2018. [Online]. Available: http://stacks.iop.org/0957-0233/29/i=6/a=065906

[4] A. R. Hoskinson and N. Hershkowitz, "Effect of finite length on the current voltage characteristic of a cylindrical Langmuir probe in a multidipole plasma chamber," Plasma Sources Science Technology, vol. 15, pp. 85-90, Feb. 2006.

[5] R. Marchand, S. Marholm, D. Darian, W. J. Miloch, and M. Mortensen, "Impact of miniaturized fixed-bias multi-needle langmuir probes on cubesats," in Proceedings of the 15th Spacecraft Charging Technology Conference. IEEE Transactions on Plasma Science, 2018.

[6] H. M. Mott-Smith and I. Langmuir, "The theory of collectors in gaseous discharges," vol. 28, pp. 727-763, 011926.

[7] J. G. Laframboise, "Theory of spherical and cylindrical langmuir probes in a collisionless, maxwellian plasma at rest," Ph.D. dissertation, University of Toronto, 1966.

[8] D. Johanning, W. Seifert, and A. Best, "Analytical density correction for cylindrical langmuir probes showing end effects," Plasma Physics and Controlled Fusion, vol. 27, no. 2, p. 159, 1985. [Online]. Available: http://stacks.iop.org/0741-3335/27/i=2/a=006

[9] R. Marchand, "PTetra, a tool to simulate low orbit satellite-plasma interaction," IEEE Transactions On Plasma Science, 2012.

[10] R. Marchand and P. A. R. Lira, "Kinetic simulation of spacecraftenvironment interaction," IEEE Transactions on Plasma Science, vol. 45 no. 4, pp. 535-554, 2017.

[11] C. Geuzaine and J.-F. Remacle, "Gmsh: A 3-d finite element mesh generator with built-in pre- and post-processing facilities," International Journal for Numerical Methods in Engineering, vol. 79, no. 11, pp. 1309-1331, 2009. [Online]. Available: http://dx.doi.org/10.1002/nme.2579

[12] E. F. Jaeger, L. A. Berry, and D. B. Batchelor, "Characteristics of a cylindrical langmuir probe of finite length,” vol. 69, pp. 6918 - 6922, 061991.

[13] E. Choiniere and B. E. Gilchrist, "Modeling long probes in flowing plasmas using kips-2d, a novel steady-state vlasov solver," 072003.

[14] R. P. Hoyt and B. M. Minor, "Remediation of radiation belts using electrostatic tether structures," vol. 2005, pp. 583 - 594, 042005.

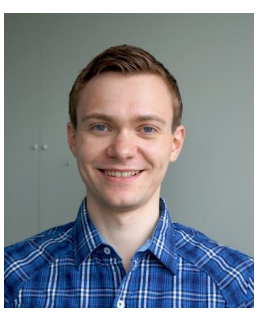

Sigvald Marholm was born in Norway 1987. He received an MSc in electrical engineering at the Norwegian University of Science and Technology (NTNU), Trondheim, Norway in 2012, and is currently pursuing a $\mathrm{PhD}$ in computational plasma physics at the University of Oslo, Norway.

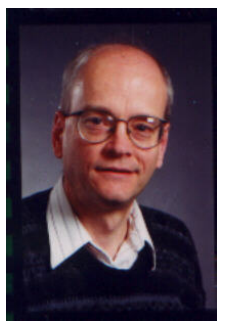

Richard Marchand is currently a Professor of Physics with the University of Alberta, Edmonton, $\mathrm{AB}$, Canada, where he carries out research in computational physics, space physics, and spacecraftenvironment interaction. 


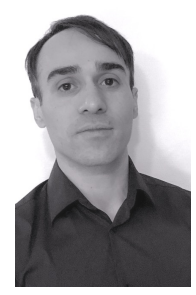

Diako Darian is a $\mathrm{PhD}$ candidate in Mechanics at the Department of Mathematics, University of Oslo, Norway. His research interests include scientific computing, ionospheric plasma, dusty plasmas, and object-plasma interactions.

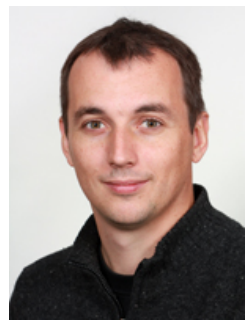

Wojciech J. Miloch received the M.Sc. degree in space and plasma physics and the Ph.D. degree from the University of Oslo, Norway, in 2006 and 2009, respectively. He is currently a professor and head of 4DSpace Strategic Research Inititative at the Department of Physics, University of Oslo. His research interests include space and astrophysical plasmas, space weather, plasma interactions with finite sized objects, such as spacecrafts, probes, or dust grains, complex plasmas and numerical modeling.

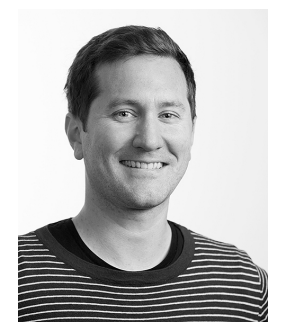

Mikael Mortensen is currently working as an Associate Professor at the Department of Mathematics, University of Oslo, where he is performing research into several aspects of turbulence and Computational Fluid Dynamics. 